Modern Physics Letters A,

(C) World Scientific Publishing Company

\title{
SAKHAROV'S INDUCED GRAVITY: A MODERN PERSPECTIVE
}

\author{
MATT VISSER* \\ Physics Department, Washington University in Saint Louis \\ Saint Louis, Missouri 63130-4899, USA
}

Received (15 April 2002)

Revised

Sakharov's 1967 notion of "induced gravity" is currently enjoying a significant resurgence. The basic idea, originally presented in a very brief 3-page paper with a total of 4 formulas, is that gravity is not "fundamental" in the sense of particle physics. Instead it was argued that gravity (general relativity) emerges from quantum field theory in roughly the same sense that hydrodynamics or continuum elasticity theory emerges from molecular physics. In this article I will translate the key ideas into modern language, and explain the various versions of Sakharov's idea currently on the market.

Contribution to the "First IUCAA Meeting on the Interface of Gravitational and Quantum Realms", held in Pune in December 2001.

To appear in Modern Physics Letters A; arXiv: gr-qc/0204062.

\section{Introduction}

Einstein gravity (general relativity) is based on two key elements: (1) pseudoRiemannian geometry (Lorentzian geometry) - the geometric notions derived from the Einstein Equivalence Principle, and (2) specific field equations for the Ricci tensor. It is the second of these elements that Sakharov addresses in developing his notion of "induced gravity". There are by now a number of versions of "induced gravity" on the market, some of which focus primarily on particle physics and others which focus primarily on (semiclassical) gravity.

To set the general framework:

- Assume you are given a Lorentzian manifold.

- Make no assumptions about the dynamics of this geometry; leave it free to flap in the breeze. Do not attempt to quantize geometry/gravity itself, but quantize everything else. (The geometry is considered as a classical background.)

* Permanent address after 1 July 2002:

School of Mathematics and Computer Science, Victoria University, PO Box 600, Wellington, New Zealand. 
- Consider one-loop quantum field theory on this manifold.

- Then at one loop the effective action is guaranteed to contain terms of the form:

$$
\int \mathrm{d}^{4} x \sqrt{-g}\left\{c_{0}+c_{1} R(g)+c_{2}\left(" R^{2} "\right)\right\} .
$$

Compare this with the standard Lagrangian for Einstein gravity

$$
\int \mathrm{d}^{4} x \sqrt{-g}\left\{-\Lambda-\frac{R(g)}{16 \pi G}+K\left(" R^{2} "\right)+\mathcal{L}_{\text {matter }}\right\} .
$$

That is: the one loop effective action automatically contains terms proportional to the cosmological constant, the Einstein-Hilbert action, plus "curvature-squared" terms.

This is the central observation, and it is extremely suggestive, but is it enough for us to recover all the interesting aspects of gravity? It is the answer to this last question that generates all the interest, and all the difficulties and subtleties.

\section{One-loop matter affects classical gravity}

Start by considering the one-loop contribution to the effective action for a (possibly non-minimally coupled) scalar field:

$$
\mathcal{S}_{g}=-\frac{1}{2} \ln \operatorname{det}\left(\Delta_{g}+m^{2}+\xi R\right)=-\frac{1}{2} \operatorname{Tr} \ln \left(\Delta_{g}+m^{2}+\xi R\right) .
$$

In Feynman diagram language this determinant represents the sum of all oneloop diagrams coupled to an arbitrary number of classical external gravitons. (Even though we have not at this stage defined gravity, we have defined the notion of geometry. To connect with particle physics language we temporarily assume that the geometry is close to flat, expand the spacetime metric as $g_{a b}=\eta_{a b}+h_{a b}$, and Fourier transform the $h_{a b}(x)$. The resulting $h_{a b}(k)$ are defined to be "external gravitons". Once you have developed this Feynman diagram picture, purely as an aid to visualization, there is no need to remain close to flat space as the determinant formula continues to make excellent sense even for highly curved and/or topologically nontrivial manifolds.) For Feynman diagrams whose only external legs are classical gravitons, particle-particle interactions will contribute only at two-loops or higher.

The determinant can be rigorously defined by a number of different techniques (e.g, zeta functions (2. B). It is somewhat more useful to adopt an explicit cutoff. Use the standard identity

$$
\ln (b / a)=\int_{0}^{\infty} \frac{\mathrm{d} x}{x}\left[e^{-a x}-e^{-b x}\right] .
$$

Now define Tr to include a trace over both spacetime and any internal indices

$$
\operatorname{Tr}[] \equiv \int \mathrm{d}^{4} x \operatorname{tr}[\mathrm{]}
$$


and adopt Schwinger's proper time formalism. ${ }^{a}$ This yields the simple expression $\mathcal{S}_{g}=\mathcal{S}_{g_{0}}+\frac{1}{2} \operatorname{Tr} \int_{0}^{\infty} \frac{\mathrm{d} s}{s}\left[\exp \left(-s\left[\Delta_{g}+m^{2}+\xi R\right]\right)-\exp \left(-s\left[\Delta_{g_{0}}+m^{2}+\xi R_{0}\right]\right)\right]$

Note that this computes the difference in the one-loop contribution to the effective that comes from comparing two different metrics ( $g$ and $g_{0}$ ) defined on the same topological manifold. ( $g_{0}$ is simply any convenient reference metric.) Since this expression is ultraviolet divergent, one should regularize with a short distance cutoff. Note that dimensionally $[s]=M^{-2}=L^{2} \equiv\left[\kappa^{-2}\right]$. The regularized but unrenormalized one-loop contribution to the effective action is then

$\mathcal{S}_{g}=\mathcal{S}_{g_{0}}+\frac{1}{2} \operatorname{Tr} \int_{\kappa^{-2}}^{\infty} \frac{\mathrm{d} s}{s}\left[\exp \left(-s\left[\Delta_{g}+m^{2}+\xi R\right]\right)-\exp \left(-s\left[\Delta_{g_{0}}+m^{2}+\xi R_{0}\right]\right)\right]$.

Now use the heat kernel expansion as $s \rightarrow 0$ :日目

$$
\exp \left(-s\left[\Delta_{g}+m^{2}+\xi R\right]\right)=\frac{\sqrt{-g}}{(4 \pi s)^{2}}\left[a_{0}(g)+a_{1}(g) s+a_{2}(g) s^{2}+\cdots\right] .
$$

The coefficients $a_{n}$ are variously denoted the Seeley-DeWitt coefficients, Hamidew coefficients, or the Minakshisundarum-Pliejel coefficients. 3 They are universal functions of the spacetime geometry, and we do not need to know their explicit form just yet. Then

$$
\begin{aligned}
\mathcal{S}_{g}= & \mathcal{S}_{g_{0}}+\frac{1}{32 \pi^{2}} \operatorname{Tr}\left\{\left[a_{0}(g)-a_{0}\left(g_{0}\right)\right] \frac{\kappa^{4}}{2}+\left[a_{1}(g)-a_{1}\left(g_{0}\right)\right] \kappa^{2}\right. \\
& \left.+\left[a_{2}(g)-a_{2}\left(g_{0}\right)\right] \ln \left(\kappa^{2} / m^{2}\right)\right\}+ \text { UV finite. }
\end{aligned}
$$

That is, the use of the heat kernel expansion has permitted us to isolate the both the form and severity of potential divergences - a result that is of course well known and is the basis, in one form or another, of essentially all work in curved-space quantum field theory. (For that matter, the occurrence of quartic, quadratic, and logarithmic divergences is quite standard in flat-space QFT.)

If we now consider Dirac/Weyl spinors, then provided there is no net chiral anomaly, we can square the Dirac operator to write

$$
\begin{aligned}
\mathcal{S} & =+\ln \operatorname{det}([\gamma \cdot D]+m) \\
& =+\frac{1}{2} \ln \operatorname{det}\left(-[\gamma \cdot D]^{2}+m^{2}\right) \\
& =+\frac{1}{2} \ln \operatorname{det}\left(\Delta+\frac{1}{4} R+m^{2}\right) .
\end{aligned}
$$

${ }^{a}$ There is nothing particularly special about Schwinger's regularization - for instance, we could just as easily use Pauli-Villars regularization. On the other hand, zeta functions or dimensional regularization are a trifle tricky because they are in a sense too powerful, and have the effect of "hiding" some of the interesting terms. 
Note the relative minus sign; in fact all fermions contribute with a relative minus sign. Summing over all particles, bose plus fermi:

$$
\begin{aligned}
\mathcal{S}_{g}= & \mathcal{S}_{g_{0}}+\frac{1}{32 \pi^{2}} \operatorname{Str}\left\{\left[a_{0}(g)-a_{0}\left(g_{0}\right)\right] \frac{\kappa^{4}}{2}+\left[a_{1}(g)-a_{1}\left(g_{0}\right)\right] \kappa^{2}\right. \\
& \left.+\left[a_{2}(g)-a_{2}\left(g_{0}\right)\right] \ln \left(\kappa^{2} / m^{2}\right)\right\}+\mathrm{UV} \text { finite. }
\end{aligned}
$$

Here we have introduced the "supertrace" operator Str, which sums over all particle species but weights fermi fields with a relative minus sign. Specifically

$$
\operatorname{Str}[] \equiv \operatorname{Tr}\left[(-)^{F}\right]
$$

Note that the usefulness of this "Str" object is not limited to supersymmetric theories, it makes perfectly good sense in arbitrary QFTs, and is simply a useful notational and bookkeeping trick.

Working from any of many standard references it is easy to see $\mathrm{A}$

$$
\begin{gathered}
a_{0}(g)=1 . \\
a_{1}(g)=k_{1} R(g)-m^{2} . \\
a_{2}(g)=k_{2} C_{a b c d} C^{a b c d}+k_{3} R_{a b} R^{a b}+k_{4} R^{2}+k_{5} \nabla^{2} R \\
-m^{2} k_{1} R(g)+\frac{1}{2} m^{4} .
\end{gathered}
$$

where the dimensionless constants $k_{i}$ depend on the particular particle species being considered. The form of these coefficients can be deduced by power counting and the fact that they must be scalar invariants of the spacetime geometry. The particular coefficients involving the $m^{2}$ and $m^{4}$ terms are easily deduced by combining a Taylor expansion of $\exp \left(-s m^{2}\right)$ with the corresponding $m=0$ Seeley-DeWitt expansion. Not all of the terms in $a_{2}$ are truly independent. Upon integration

$$
\int \sqrt{-g} a_{2}(g)=\int \sqrt{-g}\left\{k_{2}^{\prime} C_{a b c d} C^{a b c d}+k_{4}^{\prime} R^{2}-m^{2} k_{1} R(g)+\frac{1}{2} m^{4}\right\} .
$$

The $k_{5}$ term has been discarded because it is a total divergence, while the $k_{3}$ term can be eliminated using the integral formula for the 4-dimensional Euler characteristic. $k_{2}^{\prime}$ and $k_{4}^{\prime}$ are suitable linear combinations of $k_{2}, k_{3}$, and $k_{4}$. For most of the qualitative discussion below we will not need to know specific values for the $k_{i}$.

Suppose we now define str by

$$
\operatorname{Str}[] \equiv \int d^{4} x \operatorname{str}[]
$$


and proceed to unwrap the above results by collecting terms based on their geometrical form:

$$
\begin{aligned}
\mathcal{S}_{g}= & \mathcal{S}_{g_{0}}+\frac{1}{32 \pi^{2}}\left\{\operatorname{str}\left[\frac{\kappa^{4}}{2}-m^{2} \kappa^{2}+\frac{m^{4}}{2} \ln \left(\frac{\kappa^{2}}{m^{2}}\right)\right] \int \mathrm{d}^{4} x\left[\sqrt{-g}-\sqrt{-g_{0}}\right]\right. \\
& +\operatorname{str}\left[k_{1} \kappa^{2}-k_{1} m^{2} \ln \left(\frac{\kappa^{2}}{m^{2}}\right)\right] \int \mathrm{d}^{4} x\left[\sqrt{-g} R(g)-\sqrt{-g_{0}} R\left(g_{0}\right)\right] \\
& +\operatorname{str}\left[k_{2}^{\prime} \ln \left(\frac{\kappa^{2}}{m^{2}}\right)\right] \int \mathrm{d}^{4} x\left[\sqrt{-g} C^{2}(g)-\sqrt{-g_{0}} C^{2}\left(g_{0}\right)\right] \\
& \left.+\operatorname{str}\left[k_{4}^{\prime} \ln \left(\frac{\kappa^{2}}{m^{2}}\right)\right] \int \mathrm{d}^{4} x\left[\sqrt{-g} R^{2}(g)-\sqrt{-g_{0}} R^{2}\left(g_{0}\right)\right]\right\} \\
& +\mathrm{UV} \text { finite. }
\end{aligned}
$$

It is now trivial to extract the coefficients in the one-loop effective action. Define the gravitational couplings as follows

$$
\int \mathrm{d}^{4} x \sqrt{-g}\left\{-\Lambda-\frac{R(g)}{16 \pi G}+K_{2} C_{a b c d} C^{a b c d}+K_{4} R^{2}+\mathcal{L}_{\text {matter }}\right\} .
$$

Then explicitly keeping track of potential zero-loop (tree level) contributions ${ }^{b}$

$$
\begin{gathered}
\Lambda=\Lambda_{0}-\frac{1}{32 \pi^{2}} \operatorname{str}\left[\frac{\kappa^{4}}{2}-m^{2} \kappa^{2}+\frac{m^{4}}{2} \ln \left(\frac{\kappa^{2}}{m^{2}}\right)\right]+\text { UV finite. } \\
\frac{1}{G}=\frac{1}{G_{0}}-\frac{1}{2 \pi} \operatorname{str}\left[k_{1} \kappa^{2}-k_{1} m^{2} \ln \left(\frac{\kappa^{2}}{m^{2}}\right)\right]+\mathrm{UV} \text { finite. } \\
K_{2}=\left(K_{2}\right)_{0}+\frac{1}{32 \pi^{2}} \operatorname{str}\left[k_{2}^{\prime} \ln \left(\frac{\kappa^{2}}{m^{2}}\right)\right]+\mathrm{UV} \text { finite. } \\
K_{4}=\left(K_{4}\right)_{0}+\frac{1}{32 \pi^{2}} \operatorname{str}\left[k_{4}^{\prime} \ln \left(\frac{\kappa^{2}}{m^{2}}\right)\right]+\mathrm{UV} \text { finite. }
\end{gathered}
$$

These are regulated but unrenormalized one-loop expressions describing the influence of the matter sector on the gravitational couplings. We see that armed with a knowledge of the particle spectrum and the cutoff, the one-loop QFTinduced changes in the gravitational couplings are calculable. With these formulae in hand, it is now easy to go back to Sakharov's papent and see where his ideas were coming from. It is also important to note that it is at this stage that the road diverges. There are at least four main directions one can take -

- Sakharov: Demand one-loop dominance.

- Pauli: Demand one-loop finiteness.

${ }^{b}$ Of course, admitting the possibility of zero-loop contributions is anti-Sakharov in spirit, but we shall see uses for this abberant behaviour later. 
- Frolov-Fursaev: Demand one-loop calculability.

- Renormalizability: Demand one-loop calculability for certain differences.

The same one-loop formulae can then have rather different implications depending on the route you take. After a brief digression on observational bounds, let us consider the major options in turn.

\section{Experimental/Observational bounds}

Regarding the gravitational couplings, we have good experimental data on Newton's constant $G$, while for the cosmological constant we are certainly safe in asserting

$$
|8 \pi G \Lambda| \lesssim 10^{-120} M_{\text {Planck }}^{4}
$$

Current observational data indeed favours the stronger statement

$$
8 \pi G \Lambda \approx+10^{-123} M_{\text {Planck }}^{4}
$$

In contrast, constraints on the dimensionless numbers $K_{2}$ and $K_{4}$ are quite weak (amazingly weak). The linearized theory leads to a weak-field potential of Yukawa form

$$
\phi(r) \propto \frac{1}{r}-\frac{4}{3} \frac{\exp \left(-M_{\text {Planck }} r / \sqrt{K_{2}}\right)}{r}+\frac{1}{3} \frac{\exp \left(-M_{\text {Planck }} r / \sqrt{K_{4}}\right)}{r} .
$$

Note that the strength of the Yukawa terms is fixed and it is only their ranges that depend on $K_{2 / 4}$.

Negative values for $K_{2}$ and $K_{4}$ lead to objectionable tachyonic behaviour. (For instance, the static potential would not limit to the Newtonian value at large distances but would instead oscillate wildly between zero and 8/3 times the standard Newtonian value. In addition the linearized theory then exhibits various instabilities.

Positive values for $K_{2}$ and $K_{4}$ are constrained by the fact that we have directly tested the inverse square law for gravity down to approximately the millimeter scale, implying $M_{\text {Planck }} / \sqrt{K} \gtrsim 10^{-13} \mathrm{GeV}$. That is

$$
K_{2 / 4} \lesssim 10^{+64}
$$

This direct experimental bound leaves considerable maneuvering room. (Various "fifth force" experiments currently in progress are likely to improve these bounds somewhat in the not too distant future. I) Indirect arguments, based on using the $K_{4}$ term to drive cosmological inflation suggest 8

$$
K_{4} \in\left(10^{+11}, 10^{+15}\right)
$$

(Cosmology is rather insensitive to the $K_{2}$ term since the Weyl tensor is identically zero in all FRW backgrounds.) There is certainly considerable room for 
deriving better phenomenological and observational bounds on these parameters.

\section{Sakharov: one-loop dominance}

Sakharov's own interpretation of the manner in which one-loop matter influences gravity was this:

- Set all tree-level constants to zero.

- Assume one-loop physics is dominant.

- Assume most dimensionless numbers of order one.

- Assume $\kappa \approx M_{\text {Planck }}$; so there is an explicit cutoff at the Planck scale.

- Quietly agree to ignore $\Lambda, K_{2}$, and $K_{4}$.

Then the Newton constant is "induced" at one-loop. Keeping only the dominant terms in the divergence

$$
\frac{1}{G} \approx-\frac{1}{2 \pi} \operatorname{str}\left[k_{1}\right] \kappa^{2} ; \quad \operatorname{str}\left[k_{1}\right] \approx-1 .
$$

This is the key observation: an approximate formula for Newton's constant as a function of the cutoff $\kappa$ and selected features of the particle spectrum (encoded in $\left.\operatorname{str}\left[k_{1}\right]\right)$. It is this conjectured connection between Newton's constant and particle physics that has led to so much attention being given to this idea. (Over 260 citations as of February 2002.) This route certainly paints a coherent and attractive physical picture - but is there real predictive power? And is it possible to say more?

For instance, if we try to say something about the other gravitational couplings:

$$
\begin{gathered}
\Lambda \approx-\frac{1}{64 \pi^{2}} \operatorname{str}[I] \kappa^{4} ; \quad \operatorname{str}[I] \approx 0 . \\
K_{2} \approx \frac{1}{32 \pi^{2}} \operatorname{str}\left[k_{2}^{\prime}\right] \ln \left(\kappa^{2} / \mu^{2}\right) \approx 1 \\
K_{4} \approx \frac{1}{32 \pi^{2}} \operatorname{str}\left[k_{4}^{\prime}\right] \ln \left(\kappa^{2} / \mu^{2}\right) \approx 1 .
\end{gathered}
$$

Note that powers dominate over logarithms (wherever possible), and that the smallness of the observed cosmological constant is very much put in by hand. (More on this below.) In contrast $K_{2}$ and $K_{4}$ are naturally dimensionless numbers of order unity; which is certainly compatible with experiment.

\section{Pauli: QFT compensation}

A different interpretation of (some of) these formulae can be traced back all the way to Wolfgang Pauli. The key discussion is contained in his 1950 lectures 
on quantum field theory (the "Feldquantisierung"). 9 Because Pauli was working in flat space, not curved, his comments were directed towards the cosmological constant $\Lambda$ (which he rephrased in terms of the net zero-point energy of the QFT). ${ }^{c}$ Pauli did not himself consider Newton's constant $G$, but the relevant extensions are straightforward.

$\underline{\text { Step 1: }}$ To guarantee a one-loop finite result for $\Lambda$ you need:?

$$
\operatorname{str}(I)=\operatorname{str}\left(m^{2}\right)=\operatorname{str}\left(m^{4}\right)=0 .
$$

Once these finiteness constraints are enforced, the one-loop contribution to the cosmological constant is ${ }^{d}$

$$
\Lambda=\Lambda_{0}+\frac{1}{64 \pi^{2}} \operatorname{str}\left[m^{4} \ln \left(\frac{m^{2}}{\mu^{2}}\right)\right]+\text { two loops. }
$$

These finiteness constraints are a fore-runner of the idea of supersymmetry, and were certainly known to many of the developers of supersymmetry (SUSY). They give tight constraints on the particle physics content of the model; constraints that are certainly not satisfied by the standard model (SM), and certainly require "beyond the standard model" (BSM) physics. For instance, these constraints are satisfied by arbitrary SUSY theories prior to spontaneous symmetry breakdown (SSB), and by all of the non-SUSY one-loop finite QFTs.10 Note the quantity $\mu$ appearing here is simply any convenient mass scale chosen to make the argument of the logarithm dimensionless, and that $\Lambda$ is independent of $\mu$ thanks to the constraint $\operatorname{str}\left(m^{4}\right)=0$.

Step 2: Now extend Pauli's compensation idea to curved space. If you additionally assume

$$
\operatorname{str}\left(k_{1}\right)=\operatorname{str}\left(k_{1} m^{2}\right)=0,
$$

then the one-loop contribution to Newton's constant is finite and

$$
\frac{1}{G}=\frac{1}{G_{0}}-\frac{1}{2 \pi} \operatorname{str}\left[k_{1} m^{2} \ln \left(\frac{m^{2}}{\mu^{2}}\right)\right]+\text { two loops. }
$$

Note that the finiteness constraint $\operatorname{str}\left(k_{1}\right)=0$ is completely at odds with Sakharov's original version of the induced gravity proposal. Also note that this constraint guarantees that $G$ is independent of $\mu$.

${ }^{c}$ One particularly transparent way of seeing that the shift in the cosmolggical constant as formulated above is equivalent to the net zero point energy is to note that

$$
\int_{0}^{\kappa} k^{2} \sqrt{m^{2}+k^{2}} d k=\frac{\kappa^{4}}{4}+\frac{m^{2} \kappa^{2}}{4}-\frac{m^{4} \ln \left(\kappa^{2} / m^{2}\right)}{8}+\text { finite. }
$$

The left hand side is manifestly proportional to the zero point energy, while the right hand side precisely yields the one-loop shift in $\Lambda$.

${ }^{d}$ Indeed, as Pauli points out, if you now add the additional restriction $\operatorname{str}\left[m^{4} \ln \left(m^{2} / \mu^{2}\right)\right]$ then the one loop contribution to the cosmological constant (zero point energy) is not just finite, it's zero. 
Arranging these secondary finiteness constraints is considerably more complicated, the relevant coefficients being given in Table I. The vanishing of $\operatorname{str}\left(k_{1}\right)$ and $\operatorname{str}\left(k_{1} \mathrm{~m}^{2}\right)$ are now very strong constraints on the particle content. These constraints are not derivable from unbroken SUSY alone, nor does one necessarily need SUSY to satisfy these constraints. A suitable toy model has been presented by Frolov and Fursaev.11

Step 3: For the final stage, you need to additionally assume

$$
\operatorname{str}\left(k_{2}^{\prime}\right)=\operatorname{str}\left(k_{4}^{\prime}\right)=0
$$

in order to render the one-loop contributions to the " $R$ " coupling constants finite. Under this assumption

$$
K_{2 / 4}=\left(K_{2 / 4}\right)_{0}-\frac{1}{32 \pi^{2}} \operatorname{str}\left[k_{2 / 4}^{\prime} \ln \left(\frac{m^{2}}{\mu^{2}}\right)\right]+\text { two loops. }
$$

Note that $k_{2}^{\prime}$ and $k_{4}^{\prime}$ are rather messy coefficients that seem to follow no particular pattern. The quantities in Table I were generated from the corresponding results presented in Birrell and Davis 4 (No explicit toy model satisfying these Step 3 constraints in addition to Steps 1 and 2 is currently known; it appears that one would need to include higher-spin fields.)

Table 1. The coefficients $k_{0}, k_{1}, k_{2}^{\prime}$ and $k_{4}^{\prime}$.

\begin{tabular}{lcccc}
\hline Description & $k_{0}$ & $k_{1}$ & $k_{2}^{\prime}$ & $k_{4}^{\prime}$ \\
\hline Scalar (minimal) & 1 & $1 / 6$ & $1 / 120$ & $1 / 72$ \\
Scalar (conformal) & 1 & 0 & $1 / 120$ & 0 \\
Scalar (generic) & 1 & $1 / 6-\xi$ & $1 / 120$ & $(1-6 \xi)^{2} / 72$ \\
Weyl spinor & 2 & $-1 / 6$ & $-1 / 40$ & 0 \\
Dirac spinor & 4 & $-1 / 3$ & $-1 / 20$ & 0 \\
Vector $1 \oplus 0)$ & 4 & $-1 / 3$ & $7 / 60$ & $1 / 36$ \\
Spin 1 (massive) & 3 & $-1 / 2$ & $13 / 120$ & $1 / 72$ \\
Spin 1 (massless) & 2 & $-2 / 3$ & $1 / 10$ & 0 \\
\hline Chiral supermultiplet & 0 & $1 / 2$ & $1 / 24$ & $1 / 36$ \\
Vector supermultiplet (massless) & 0 & $1 / 2$ & $1 / 8$ & 0 \\
Vector supermultiplet (massive) & 0 & 0 & $5 / 24$ & $1 / 24$ \\
\hline
\end{tabular}

Total contributions to the $k_{i}$ summed over spin states for low-spin particles. (Here $k_{0}=\operatorname{tr}[I]$ counts the number of spin states.) For supermultiplets the net value, including the effect of the minus sign for fermions, is reported.

The overall conclusion is that one can keep all the one-loop effects of matter on the gravity sector finite, at the cost of very strong constraints on the particle spectrum, requiring rather specific BSM physics. But maybe the price paid is too high? Indeed Pauli finishes his own comments with the observation:

These requirements are so extensive that it is rather improbable that they are satisfied in reality. 
(And Pauli was only concerned with Step 1; the particle masses and their influence on the cosmological constant. His technique was completely unable to even begin addressing Newton's constant and $K_{2 / 4}$ as influenced by the $k_{i}$ 's and their constraints.)

\section{Frolov-Fursaev: one-loop calculability}

Suppose we boldly assume both Pauli compensation and Sakharov one-loop dominance. That is, assume all finiteness constraints:

$$
\begin{gathered}
\operatorname{str}(I)=\operatorname{str}\left(m^{2}\right)=\operatorname{str}\left(m^{4}\right)=0 . \\
\operatorname{str}\left(k_{1}\right)=\operatorname{str}\left(k_{1} m^{2}\right)=0 . \\
\operatorname{str}\left(k_{2}^{\prime}\right)=\operatorname{str}\left(k_{4}^{\prime}\right)=0 .
\end{gathered}
$$

And in addition, assume all zero-loop (tree level) coefficients are zero. This is (essentially) the Frolov-Fursaev variant of Sakharov's proposal.11 (Note that in the specific model they implement, Frolov and Fursaev do not impose finiteness/calculability on $K_{2}$ and $K_{4}$, and only impose the first two lines of constraints.) Then $\Lambda, G$, and $K_{2 / 4}$ are one-loop calculable:

$$
\begin{gathered}
\Lambda=+\frac{1}{64 \pi^{2}} \operatorname{str}\left[m^{4} \ln \left(\frac{m^{2}}{\mu^{2}}\right)\right]+\text { two loops. } \\
\frac{1}{G}=-\frac{1}{2 \pi} \operatorname{str}\left[k_{1} m^{2} \ln \left(\frac{m^{2}}{\mu^{2}}\right)\right]+\text { two loops. } \\
K_{2 / 4}=\frac{1}{32 \pi^{2}} \operatorname{str}\left[k_{2 / 4}^{\prime} \ln \left(\frac{m^{2}}{\mu^{2}}\right)\right]+\text { two loops. }
\end{gathered}
$$

In contrast to Sakharov's original idea, the cutoff has now disappeared. Given a particle spectrum that satisfies the finiteness constraints, the one-loop induced gravitational couplings are unambiguous. This is a very interesting proposal, but it requires very tight interlocking constraints (extended Pauli compensation) on the particle spectrum to permit it to work.

\section{Renormalizability: invariance constraints}

Suppose we want to be a little less ambitious, perhaps the interlocking constraints of Pauli compensation seem excessive, perhaps Sakharov's version of one-loop dominance with an explicit cutoff seems inelegant: Is there anything interesting you can say just using "standard" renormalization theory?

By this I mean that we are asking that particle physics be at least oneloop renormalizable in an arbitrary classical background gravitational field note that I am not placing any constraints on renormalizability of gravity itself. (Indeed at this stage "gravity" is a purely descriptive statement about the existence of curved spacetime and has no dynamics.) Instead I am imposing the 
much weaker condition that particle physics does not violently damage classical Einstein gravity. The very fact that both particle physics and classical Einstein gravity work reasonably well on the surface of planet Earth is good evidence that this is a quite reasonable physical demand.

Then renormalization in the gravity sector does indeed allow you to absorb the one-loop divergences into the zero-loop bare quantities - but you are allowed to do this once and once only. Once you have renormalized, you are not allowed to change your mind about how big an infinity you dump into the zero-loop bare quantities.

In particular: This means that QFTs can naturally be assigned to equivalence classes under renormalization of the gravity sector. As one tunes the QFT coupling constants, so that the QFT goes through a phase transition or $\mathrm{SSB}$, then the masses in the particle spectrum change, (and the very content of the particle spectrum changes due to Higgs particles being eaten by gauge bosons). Then gravitationally-equivalent QFTs are defined by demanding that the coefficients of $\kappa^{4}, \kappa^{2}$, and $\log \left(\kappa^{2} / \mu^{2}\right)$ in the regulated effective action are not allowed to change. (Otherwise you are doing the equivalent of changing the zero of energy in the middle of the calculation, or worse.) That is, as the QFT goes through a phase transition or SSB, some specific quantities should be held invariant, and in particular:

$$
\begin{gathered}
\delta \operatorname{str}(I)=\delta \operatorname{str}\left(m^{2}\right)=\delta \operatorname{str}\left(m^{4}\right)=0 . \\
\delta \operatorname{str}\left(k_{1}\right)=\delta \operatorname{str}\left(k_{1} m^{2}\right)=0 . \\
\delta \operatorname{str}\left(k_{2}^{\prime}\right)=\delta \operatorname{str}\left(k_{4}^{\prime}\right)=0 .
\end{gathered}
$$

Note that as long as one is working strictly in flat space, the invariance constraints involving the $\operatorname{str}\left(k_{i}\right)$ would never appear. The invariance constraints involving $\operatorname{str}\left(m^{n}\right)$ appear only if you take the net zero of energy seriously and it has been traditional in flat-space QFT to simply ignore the overall net zero and only ask questions about excitations.

Some of these constraints are automatically satisfied - (1) Changing the coupling constants, even if one induces SSB or a phase transition does not change the net number of fermions or bosons so $\delta \operatorname{str}[I]=0$ automatically. (2) As one goes through SSB a Higgs scalar is "eaten" by a gauge boson which becomes massive. However $k_{1}$ for a massive vector is calculated by adding $k_{1}$ for a minimally coupled scalar to $k_{1}$ for a massless vector. Other particles may acquire mass but do not change their intrinsic character. Thus $\delta \operatorname{str}\left[k_{1}\right]=0$ provided all the Higgs particles are minimally coupled scalars $[\xi=0]$. (3) The same argument applies to $\delta \operatorname{str}\left[k_{2}^{\prime}\right]$ and $\delta \operatorname{str}\left[k_{4}^{\prime}\right]$, without now any constraint on the curvature coupling. Thus the only nontrivial invariance constraints are

$$
\delta \operatorname{str}\left(m^{2}\right)=\delta \operatorname{str}\left(m^{4}\right)=\delta \operatorname{str}\left(k_{1} m^{2}\right)=0 .
$$


We can view these invariance constraints as demanding that for gravitationallyequivalent QFTs there are strong correlations between mass changes in the fermi and bose sectors. It is far from trivial to satisfy these constraints, but it is certainly much easier to do this than to satisfy the much stronger (extended) Pauli finiteness constraints. Provided these invariance constraints are satisfied then the gravitational couplings have finite changes as one varies the parameters that are one-loop calculable:

$$
\begin{gathered}
\delta \Lambda=+\frac{1}{64 \pi^{2}} \delta \operatorname{str}\left[m^{4} \ln \left(\frac{m^{2}}{\mu^{2}}\right)\right]+\text { two loops. } \\
\delta\left(\frac{1}{G}\right)=-\frac{1}{2 \pi} \delta \operatorname{str}\left[k_{1} m^{2} \ln \left(\frac{m^{2}}{\mu^{2}}\right)\right]+\text { two loops. } \\
\delta K_{2 / 4}=\frac{1}{32 \pi^{2}} \delta \operatorname{str}\left[k_{2 / 4}^{\prime} \ln \left(\frac{m^{2}}{\mu^{2}}\right)\right]+\text { two loops. }
\end{gathered}
$$

In this case, if you know the change in the particle spectrum, and can verify that it satisfies the invariance constraints, then you can calculate (at one-loop) finite changes in $\Lambda, G$, and $K$. (Note these changes are $\mu$-independent.)

To reiterate: these invariance constraints are not satisfied by the standard model (SM) as you modify the Higgs couplings or the Yukawa couplings. That is: In the SM coupled to classical gravity (the curved-space SM), even after you renormalize, all of the gravitational couplings, $\Lambda, G$, and $K$, are "unstable" in the sense that they still suffer infinite shifts whenever the SM coupling constants vary infinitesimally. This is one particularly acute version of the "cosmological constant problem" (itself a variant of the "hierarchy problem") demonstrating why "beyond the standard model" (BSM) physics is essential. It is not just $\Lambda$ that is at risk, the Newton constant $G$ is also subject to potentially disastrous sensitivity to the coupling constants. Even if BSM physics patches up the invariance constraints, one should still expect finite shifts in $\Lambda, G$, and $K$. Can these be made compatible with experiment/observation?

Order unity changes in $K_{2 / 4}$ are not a problem since the experimental bounds on these constants are so poor. Assuming our invariance constraints, uncertainties in the Newton constant due to uncertainties in the coupling constants are of fractional order $\Delta g\left(M_{E W} / M_{\text {Planck }}\right)^{2} \approx \Delta g 10^{-34}$, and unlikely to cause immediate concern. It is changes in the cosmological constant that still prove challenging. It is still distressingly easy to generate a "large" shift in the cosmological constant. (That is, "large" as far as cosmology/astrophysics is concerned, consisting of numerous partially-cancelling terms each of which is individually $O\left(M_{E W}^{4}\right)$; this is "small" from a particle physics perspective but would still seem to require some fine tuning.)

Suppose now that instead of comparing two different QFTs with different couplings we want to consider a single definite QFT as a function of renormalization scale. Then the the discussion is better rephrased in terms of $\beta$-functions 
and anomalous dimensions ( $\gamma$-functions). Define, in the usual particle physics fashion

$$
\beta_{g} \equiv \mu \frac{\partial g}{\partial \mu} ; \quad \gamma_{m} \equiv \frac{\mu}{m} \frac{\partial m}{\partial \mu} .
$$

Then one can define differential versions of the invariance constraints:

$$
\operatorname{str}\left[\begin{array}{ll}
\gamma_{m} & m^{2}
\end{array}\right]=\operatorname{str}\left[\begin{array}{ll}
\gamma_{m} & m^{4}
\end{array}\right]=\operatorname{str}\left[\begin{array}{lll}
k_{1} & \gamma_{m} & m^{2}
\end{array}\right]=0,
$$

though the physics interpretation is now rather different. These differential versions of the invariance constraints greatly simplify the gravitational $\beta$-functions so that

$$
\begin{aligned}
\beta_{\Lambda} & =-\frac{1}{32 \pi^{2}}\left\{\operatorname{str}\left[m^{4}\right]-2 \operatorname{str}\left[\gamma_{m} m^{4} \ln \left(\frac{m^{2}}{\mu^{2}}\right)\right]\right\}+\text { two loops. } \\
\beta_{1 / G} & =+\frac{1}{\pi}\left\{\operatorname{str}\left[k_{1} m^{2}\right]-\operatorname{str}\left[k_{1} \gamma_{m} m^{2} \ln \left(\frac{m^{2}}{\mu^{2}}\right)\right]\right\}+\text { two loops. } \\
\beta_{K_{2 / 4}} & =+\frac{1}{16^{2} \pi}\left\{\operatorname{str}\left[k_{2 / 4}^{\prime}\right]-\operatorname{str}\left[k_{2 / 4}^{\prime} \gamma_{m}\right]\right\}+\text { two loops. }
\end{aligned}
$$

(Because of the constraints, the apparent explicit $\mu$ dependence is again an illusion.) If we now move into a region where the particle masses are approximately constant $\gamma_{m} \approx 0$, explicit integration of the beta functions is trivial: the gravitational couplings exhibit standard logarithmic running. Indeed the one-loop regulated formulae for $\Lambda, G$, and $K_{2 / 4}$ now lead to:

$$
\begin{gathered}
\Lambda(\mu)=\Lambda\left(\mu_{0}\right)-\frac{1}{64 \pi^{2}} \operatorname{str}\left[m^{4}\right] \ln \left(\frac{\mu^{2}}{\mu_{0}^{2}}\right)+\text { two loops. } \\
\frac{1}{G(\mu)}=\frac{1}{G\left(\mu_{0}\right)}+\frac{1}{2 \pi} \operatorname{str}\left[k_{1} m^{2}\right] \ln \left(\frac{\mu^{2}}{\mu_{0}^{2}}\right)+\text { two loops. } \\
K_{2 / 4}(\mu)=K_{2 / 4}\left(\mu_{0}\right)+\frac{1}{32 \pi^{2}} \operatorname{str}\left[k_{2 / 4}^{\prime}\right] \ln \left(\frac{\mu^{2}}{\mu_{0}^{2}}\right)+\text { two loops. }
\end{gathered}
$$

These formulae should be understood with all the usual particle physics caveats regarding the physical interpretation of the renormalization point $\mu$. They are useful shorthand for estimating the strength of the dressed gravitational vertex (classical external gravity dressed with one-loop matter) in scattering experiments at an energy scale $\mu$. One should not blindly insert these running couplings into the classical field equations.

All in all, it is clear that even quite ordinary straightforward applications of usual ideas of renormalizability have quite powerful implications in curved spacetime - not only can we extract information regarding classical gravity, but we can turn around and use classical gravity to suggest interesting constraints on the particle spectrum. 


\section{Variants}

You can also construct variations on these four main themes by mixing and matching. For instance one attractive variant of the original Sakharov approach is to improve its treatment of the cosmological constant by using the Pauli finiteness constraints to deal with $\Lambda$, while reserving Sakharov's cutoff approach for the Newton constant $G$ and $K_{2 / 4}$.

Similarly a variant on one-loop calculability (which is closer in detail, though not in spirit, to what Frolov and Fursaev actually do) is to use combined Pauli finiteness and Sakharov one-loop dominance on $\Lambda$ and $G$, but take care of $K_{2 / 4}$ using standard renormalization theory.

There are also variants of induced gravity in $1+1$ and $2+1$ dimensions, 22,13 where the curvature-squared terms quietly drop out. In contrast in $5+1$ dimensions (or higher) one would need to keep track of curvature-cubed terms (or higher) as well. Other possibilities abound, such as the inclusion of external dilaton fields or string-inspired moduli fields.

\section{Summary and Discussion}

One point that all approaches agree on is that one-loop matter effects lead to shifts in $\Lambda, G$, and $K$. Depending on your choices you can interpret these shifts using either -

- Sakharov: one-loop dominance.

- Pauli: one-loop finiteness.

- Frolov-Fursaev: one-loop calculable.

- Renormalization: calculable one-loop changes.

Whatever your viewpoint in this regard, the key point is this: Even if Einstein gravity is not there at zero loops, it will automatically be generated at one-loop.

Thus we have seen how Sakharov's ideas have been very fruitful - and how they naturally lead in a number of different directions. There are still many interesting questions to answer just at the level of QFT and particle phenomenology. (Without requiring any particularly radical reassessment of our world-view.) In particular the finiteness constraints (or even the much weaker invariance constraints) place very interesting restrictions on the allowable spectrum of particles in BSM physics.

But Sakharov also teaches us a lot about gravity: Suppose you have a manifold that is "free to flap in the breeze", on which you proceed to construct a QFT. Then the geometry of that manifold serves as a classical external field, and the effective action is a function of that geometry. Extremizing the effective action in the usual manner, because it automatically contains the classical gravitational action, automatically leads to semiclassical quantum gravity, at 
least at the one-loop level. (By semiclassical gravity I mean that the classical Einstein equations, plus curvature-squared corrections, are coupled to the expectation value of the quantum stress-energy tensor for all the quantized matter fields.)

The important point here is that gravity was never put into the quantum theory, and gravity was never quantized in any way shape or form. Nevertheless, classical gravity (meaning the inverse square law implicit in the Einstein equations and Einstein-Hilbert action) automatically emerges in the semiclassical limit. If you couple this with the observation that the only actual experiments we can (currently) perform with gravity also inhabit this same semiclassical realm, is there any real need to quantize gravity itself?

Most physicists would still answer this question in the affirmative - but then Sakharov's ideas still have considerable impact: If we consider any candidate theory for quantum gravity (brane models, quantum geometry, lattice quantum gravity) then Sakharov's scenario tells us that trying to derive the inverse square law from first principles is not the difficult step. Deriving the inverse square law, and all of Einstein gravity is in fact automatic once you have demonstrated the existence of Lorentzian manifolds - reasonably large reasonably flat arenas on which to set up a low-energy QFT.

\section{Acknowledgments}

I wish to thank Larry Yaffe for helpful comments and criticisms. This research was supported by the US DOE.

1. A.D. Sakharov "Vacuum Quantum Fluctuations In Curved Space And The Theory Of Gravitation", Sov. Phys. Dokl. 12 (1968) 1040 [Dokl. Akad. Nauk Ser. Fiz. 177 (1968) 70]. Reprinted in Gen. Rel. Grav. 32 (2000) 365-367.

2. J. S. Dowker and R. Critchley, "Effective Lagrangian And Energy Momentum Tensor In De Sitter Space", Phys. Rev. D 13 (1976) 3224.

S. W. Hawking, "Zeta Function Regularization Of Path Integrals In Curved SpaceTime", Commun. Math. Phys. 55 (1977) 133.

3. S. Blau, M. Visser and A. Wipf, "Zeta Functions And The Casimir Energy", Nucl. Phys. B 310 (1988) 163.

4. N. D. Birrell and P. C. Davies, "Quantum Fields In Curved Space", Cambridge University Press, (1982).

5. K. S. Stelle, "Renormalization Of Higher Derivative Quantum Gravity", Phys. Rev. D 16 (1977) 953.

"Classical Gravity With Higher Derivatives", Gen. Rel. Grav. 9 (1978) 353.

6. G. T. Horowitz and R. M. Wald, "Dynamics Of Einstein's Equation Modified By A Higher Order Derivative Term", Phys. Rev. D 17 (1978) 414.

7. E. G. Adelberger, "Sub-millimeter tests of the gravitational inverse square law", arXiv:hep-ex/0202008

8. M. B. Mijic, M. S. Morris and W. M. Suen, "The R**2 Cosmology: Inflation Without A Phase Transition", Phys. Rev. D 34 (1986) 2934.

"Initial Conditions For The R + Epsilon R**2 Cosmology", Phys. Rev. D 39 (1989) 
1496.

9. W. Pauli, Pauli Lectures on Physics: Vol 6, Selected Topics in Field Quantization, MIT Press, 1971 (editor C.P. Enz).

(Translation of "Feldquantisierung" 1950-51; see esp. p. 33 of the English translation.)

10. Finite QFTs are most commonly investigated assuming supersymmetry, see for instance:

P. S. Howe, K. S. Stelle and P. C. West, "A Class Of Finite Four-Dimensional Supersymmetric Field Theories", Phys. Lett. B 124 (1983) 55.

C. Lucchesi and G. Zoupanos, "All-order Finiteness in N=1 SYM Theories: Criteria and Applications", Fortsch. Phys. 45 (1997) 129 arXiv:hep-ph/9604216.

W. Siegel, "Fields", arXiv:hep-th/9912205. (See esp. VIII A 5-6, pp 425-427.)

K. Yoshioka, "Finite SUSY GUT revisited", Phys. Rev. D 61 (2000) 055008 arXiv:hep-ph/9705449.

There are also a number of attempts at retaining finiteness while relaxing the supersymmetry constraint, see for instance:

W. Lucha and M. Moser, "Clifford algebras in finite quantum field theories. I: Irreducible Yukawa finiteness condition", Int. J. Mod. Phys. A 13 (1998) 2047 arXiv:hep-th/9702137.

V. E. Lemes, M. S. Sarandy, S. P. Sorella, O. S. Ventura and L. C. Vilar, "An algebraic criterion for the ultraviolet finiteness of quantum field theories", J. Phys. A 34 (2001) 9485 arXiv:hep-th/0103110.

P. Grandits, "On Nonsupersymmetric Finite Quantum Field Theories", Int. J. Mod. Phys. A 10 (1995) 1507.

P. Grandits, "No go theorems for nonsupersymmetric finite quantum field theories", Mod. Phys. Lett. A 9 (1994) 1093.

H. Skarke, "Renormalization group flow in a general gauge theory", Phys. Lett. B 336 (1994) 32 arXiv:hep-th/9407086.

D. R. Jones, L. Mezincescu and Y. P. Yao, "Soft Breaking Of Two Loop Finite N=1 Supersymmetric Gauge Theories", Phys. Lett. B 148 (1984) 317.

For the purposes of the present discussion, remember that one-loop finiteness is quite sufficient.

11. V. P. Frolov, D. V. Fursaev and A. I. Zelnikov, "Black Hole Statistical Mechanics And Induced Gravity", Nucl. Phys. Proc. Suppl. 57 (1997) 192.

V. P. Frolov and D. V. Fursaev, "Statistical mechanics on axially-symmetric spacetimes with the Killing horizon and entropy of rotating black holes in induced gravity", Phys. Rev. D 61 (2000) 024007 arXiv:gr-qc/9907046.

V. Frolov and D. Fursaev, "Black hole entropy in induced gravity: Reduction to 2D quantum field theory on the horizon", Phys. Rev. D 58 (1998) 124009 arXiv:hepth/9806078.

V. P. Frolov and D. V. Fursaev, "Thermal fields, entropy, and black holes", Class. Quant. Grav. 15, 2041 (1998) arXiv:hep-th/9802010.

V. P. Frolov and D. V. Fursaev, "Plenty of nothing: Black hole entropy in induced gravity", arXiv:hep-th/9705207.

V. P. Frolov and D. V. Fursaev, "Mechanism of generation of black hole entropy in Sakharov's induced gravity", Phys. Rev. D 56 (1997) 2212 arXiv:hep-th/9703178. V. P. Frolov, D. V. Fursaev and A. I. Zelnikov, "Statistical origin of black hole entropy in induced gravity", Nucl. Phys. B 486 (1997) 339 arXiv:hep-th/9607104.

12. V. Frolov, D. Fursaev, J. Gegenberg and G. Kunstatter, "Thermodynamics and statistical mechanics of induced Liouville gravity", Phys. Rev. D 60 (1999) 024016 
arXiv:hep-th/9901087.

13. V. P. Frolov and D. V. Fursaev, "Statistical mechanics of charged black holes in induced Einstein-Maxwell gravity", Phys. Rev. D 61 (2000) 064010 arXiv:hepth/9910006. 